Pacific

Journal of

Mathematics

\title{
BERGMAN THEORY OF CERTAIN GENERALIZED HARTOGS TRIANGLES
}

LUKE D. EDHOLM 


\title{
BERGMAN THEORY OF CERTAIN GENERALIZED HARTOGS TRIANGLES
}

\author{
LUKE D. EDHOLM
}

\begin{abstract}
The Bergman theory of domains $\left\{\left|z_{1}\right|^{\gamma}<\left|z_{2}\right|<1\right\}$ in $\mathbb{C}^{2}$ is studied for certain values of $\gamma$, including all positive integers. For such $\gamma$, we obtain a closed form expression for the Bergman kernel $\mathbb{B}_{\gamma}$. With these formulas, we make new observations relating to the Lu Qi-Keng problem and analyze the boundary behavior of $\mathbb{B}_{\gamma}(z, z)$.
\end{abstract}

\section{Introduction}

For a domain $\Omega \subset \mathbb{C}^{n}$, the Bergman space is the set of square-integrable, holomorphic functions on $\Omega$. The Bergman kernel is a reproducing integral kernel on the Bergman space that is indispensable to the study of holomorphic functions in several complex variables. The purpose of this paper is to understand Bergman theory for a class of bounded, pseudoconvex domains in $\mathbb{C}^{2}$. Define the generalized Hartogs triangle of exponent $\gamma>0$ to be the domain

$$
\mathbb{H}_{\gamma}=\left\{\left(z_{1}, z_{2}\right) \in \mathbb{C}^{2}:\left|z_{1}\right|^{\gamma}<\left|z_{2}\right|<1\right\} .
$$

$\mathbb{W}_{1}$ is the "classical" Hartogs triangle, a well-known pseudoconvex domain with nontrivial Nebenhülle. When $\gamma>1$, we call $\mathbb{H}_{\gamma}$ a fat Hartogs triangle, and when $0<\gamma<1$, we call $\mathbb{U}_{\gamma}$ a thin Hartogs triangle. Our main results are the following two computations.

Theorem 1.2. Let $s:=z_{1} \bar{w}_{1}, t:=z_{2} \bar{w}_{2}$, and $k \in \mathbb{Z}^{+}$. The Bergman kernel for the fat Hartogs triangle $\mathbb{H}_{k}$ is given by

$$
\mathbb{B}_{k}(z, w)=\frac{p_{k}(s) t^{2}+q_{k}(s) t+s^{k} p_{k}(s)}{k \pi^{2}(1-t)^{2}\left(t-s^{k}\right)^{2}},
$$

where $p_{k}$ and $q_{k}$ are the polynomials

$$
p_{k}(s)=\sum_{l=1}^{k-1} l(k-l) s^{l-1}, \quad q_{k}(s)=\sum_{l=1}^{k}\left(l^{2}+(k-l)^{2} s^{k}\right) s^{l-1} .
$$

MSC2010: 32A25, 32A36, 32W05.

Keywords: Bergman kernel, fat Hartogs triangle, Bell's transformation formula, Lu Qi-Keng. 
Theorem 1.4. Let $s=z_{1} \bar{w}_{1}, t=z_{2} \bar{w}_{2}$, and $k \in \mathbb{Z}^{+}$. The Bergman kernel for the thin Hartogs triangle $\mathbb{W}_{1 / k}$ is given by

$$
\mathbb{B}_{1 / k}(z, w)=\frac{t^{k}}{\pi^{2}(1-t)^{2}\left(t^{k}-s\right)^{2}} .
$$

There has been an extensive amount of research devoted to understanding Bergman kernels of various classes of domains, and there are several instances in which explicit formulas for the kernel have been obtained. The most common method involves summing an infinite series, which is done in [D'Angelo 1978; 1994; Park 2008]. In [Boas et al. 1999], explicit formulas for the Bergman kernel are produced using other techniques which avoid infinite series altogether. But these situations are exceptional, and in most cases it is impossible to express the Bergman kernel in closed form.

Despite the difficulty of producing explicit formulas, powerful estimates on the Bergman kernel have been given for many classes of pseudoconvex domains. Fefferman [1974] develops an asymptotic expansion of the kernel on smoothly bounded, strongly pseudoconvex domains in $\mathbb{C}^{n}$. Useful estimates also exist for large classes of smoothly bounded, weakly pseudoconvex domains. See [Catlin 1989; McNeal 1989; 1994; Nagel et al. 1989] for some of the principal results on finite type domains, and [Fu 2014] for domains with locally smooth boundaries and constant Levi-rank.

At present, there are no general theorems about the behavior of the Bergman kernel on pseudoconvex domains near unsmooth boundary points, which adds to the intrigue of Theorems 1.2 and 1.4. Each generalized Hartogs triangle defined by (1.1) has two very different kinds of boundary irregularities: the "corner points" which occur at the intersection of the two bounding real hypersurfaces, and the origin singularity, near which $b \mathbb{W}_{k}$ cannot be expressed as the graph of a continuous function.
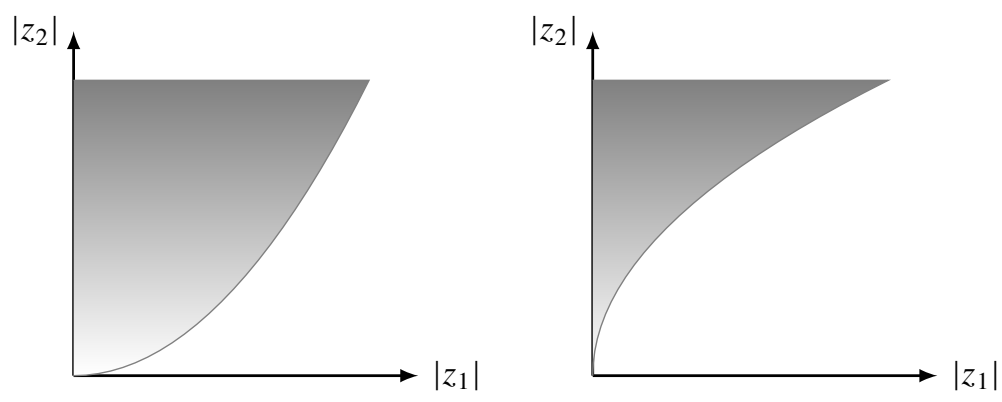

This is one of several recent papers to study holomorphic function theory on domains with similar kinds of boundary singularities. Chakrabarti and Shaw [2013] investigate the Sobolev regularity of the $\bar{\partial}$-equation on the classical Hartogs triangle. 
Chakrabarti and Zeytuncu [2016] study the $L^{p}$-mapping properties of the Bergman projection on the classical Hartogs triangle, and Chen [2013] studies $L^{p}$-mapping of the Bergman projection on analogous domains in higher dimensions. Zapałowski [2016] characterizes proper maps between generalizations of the Hartogs triangle in $\mathbb{C}^{n}$. This author and McNeal investigate the Bergman projection on fat Hartogs triangles in [Edholm and McNeal 2016]. It can be hoped that by understanding the Bergman theory on example domains with boundary singularities such as $\mathbb{H}_{\gamma}$, we can gain deeper insight into the situation on more general domains.

\section{Preliminaries}

Bergman theory. Here we highlight some basic facts about Bergman theory that are used throughout this paper. See [Krantz 1992] for a more detailed treatment. If $\Omega \subset \mathbb{C}^{n}$ is a domain, let $O(\Omega)$ denote the holomorphic functions on $\Omega$. The standard $L^{2}$ inner product is denoted by

$$
\langle f, g\rangle=\int_{\Omega} f \cdot \bar{g} d V
$$

where $d V$ denotes Lebesgue measure on $\mathbb{C}^{n} . L^{2}(\Omega)$ denotes the measurable functions $f$ such that $\langle f, f\rangle=\|f\|^{2}<\infty$. We define the Bergman space $A^{2}(\Omega):=$ $\mathcal{O}(\Omega) \cap L^{2}(\Omega)$.

$A^{2}(\Omega)$ is a Hilbert space with inner product (2.1), and for all $z \in \Omega$, the evaluation functional $\mathrm{ev}_{z}: f \mapsto f(z)$ is continuous. Therefore, the Riesz representation theorem guarantees the existence of a function $\mathbb{B}_{\Omega}: \Omega \times \Omega \rightarrow \mathbb{C}$ satisfying

$$
f(z)=\int_{\Omega} \mathbb{B}_{\Omega}(z, w) f(w) d V(w), \quad f \in A^{2}(\Omega) .
$$

We call $\mathbb{B}_{\Omega}$ the Bergman kernel, and when context is clear we may omit the subscript. In addition to reproducing functions in the Bergman space via (2.2), the Bergman kernel is conjugate symmetric and for each fixed $w \in \Omega, \mathbb{B}(\cdot, w) \in A^{2}(\Omega)$.

Given an orthonormal Hilbert space basis $\left\{\phi_{\alpha}\right\}_{\alpha \in \mathscr{A}}$ for $A^{2}(\Omega)$, the Bergman kernel is given by the formula

$$
\mathbb{B}(z, w)=\sum_{\alpha \in \mathscr{A}} \phi_{\alpha}(z) \overline{\phi_{\alpha}(w)},
$$

which is independent of the choice of the basis.

Finally, the Bergman kernel transforms under biholomorphisms in the following way: Let $F: \Omega \rightarrow \widetilde{\Omega}$ be a biholomorphic map of domains in $\mathbb{C}^{n}$. Then

$$
\mathbb{B}_{\Omega}(z, w)=\operatorname{det} F^{\prime}(z) \cdot \mathbb{B}_{\widetilde{\Omega}}(F(z), F(w)) \cdot \overline{\operatorname{det} F^{\prime}(w)} .
$$


The Bergman kernel of $\mathbb{H}_{1}$. The formula for the Bergman kernel of the classical Hartogs triangle has been known for quite some time, at least since Bremermann's [1955] paper. Following the spirit of Bremermann's argument, we use formula (2.4) to compute $\mathbb{B}_{\mathbb{H}_{1}}$. The map given by $\psi\left(z_{1}, z_{2}\right)=\left(z_{1} / z_{2}, z_{2}\right)$ is a biholomorphism of $\mathbb{H}_{1}$ onto $D \times D^{*}$, where $D$ is the unit disc and $D^{*}$ is the punctured disc. It's easy to see that the Bergman kernel of $D \times D^{*}$ is the same as that of $D \times D$, which is well known and given by

$$
\mathbb{B}_{D \times D}(z, w)=\frac{1}{\pi^{2}\left(1-z_{1} \bar{w}_{1}\right)^{2}\left(1-z_{2} \bar{w}_{2}\right)^{2}}=\mathbb{B}_{D \times D^{*}}(z, w) .
$$

Seeing that $\operatorname{det} \psi^{\prime}(z)=1 / z_{2},(2.4)$ says

$$
\mathbb{B}_{\mathbb{H}_{1}}(z, w)=\frac{z_{2} \bar{w}_{2}}{\pi^{2}\left(1-z_{2} \bar{w}_{2}\right)^{2}\left(z_{2} \bar{w}_{2}-z_{1} \bar{w}_{1}\right)^{2}} .
$$

Because of this computation, Theorems 1.2 and 1.4 only need to be proved for integers $k \geq 2$. Note the polynomial $p_{1}(s)$ in (1.3) is vacuously equal to 0 .

Distance to the boundary and asymptotic growth rates. The following notation will be used in and around Theorem 4.9. Given any $z \in \Omega$, define the distance to the boundary of $\Omega$ by the function

$$
\delta_{\Omega}(z):=\min \{\|z-\zeta\|: \zeta \in b \Omega\},
$$

where $\|\cdot\|$ denotes Euclidean distance. When the context is clear, we may omit the subscript. We will also use the following notation to write inequalities. If $A$ and $B$ are functions depending on several variables, write $A \lesssim B$ to mean that there is a constant $K>0$, independent of relevant variables, such that $A \leq K \cdot B$. The independence of which variables will be clear in context. Also write $A \approx B$ to mean that $A \lesssim B \lesssim A$.

\section{Bell's transformation rule and derivation of the kernel}

Equation (2.4) says that the Bergman kernels of two biholomorphic domains are related by a simple formula. But applications of this transformation rule remain limited by the fact that it's rare to expect two domains in $\mathbb{C}^{n}$ to be biholomorphic. There is, however, a more general version of this transformation rule. Bell [1982] proves a generalization which applies whenever we have two domains and a proper holomorphic map from one onto the other. The statement of this more general transformation rule appears below, and it will be essential to our proof of Theorem 1.2.

Recall the classical fact that any holomorphic, proper map of $\Omega$ onto $\widetilde{\Omega}$ is necessarily a branched covering of finite order. 
Theorem 3.1 (Bell's transformation rule). Let $\Omega$ and $\widetilde{\Omega}$ be domains in $\mathbb{C}^{n}$ with respective Bergman kernels $\mathbb{B}$ and $\widetilde{\mathbb{B}}$, and suppose $\phi$ is a proper holomorphic map of order $k$ from $\Omega$ onto $\widetilde{\Omega}$. Let $u:=\operatorname{det}\left[\phi^{\prime}\right]$, and let $\Phi_{1}, \Phi_{2}, \ldots, \Phi_{k}$ be the branch inverses of $\phi$ defined locally on $\widetilde{\Omega}-V$, where $V:=\{\phi(z): u(z)=0\}$. Finally, write $U_{j}:=\operatorname{det}\left[\Phi_{j}^{\prime}\right]$. Then

$$
u(z) \widetilde{\mathbb{B}}(\phi(z), w)=\sum_{j=1}^{k} \mathbb{B}\left(z, \Phi_{j}(w)\right) \overline{U_{j}(w)} .
$$

We're now ready to compute the Bergman kernel of fat Hartogs triangles with integer exponents. For the rest of this paper, we'll denote the Bergman kernel of $\mathbb{U}_{\gamma}$ by $\mathbb{B}_{\gamma}$.

Proof of Theorem 1.2. First we need to define the map $\phi$ and its local inverses $\Phi_{1}, \ldots, \Phi_{k}$. For each integer $k \geq 2$, the function $\phi: \mathbb{H}_{1} \rightarrow \mathbb{U}_{k}$ given by

$$
\phi(z)=\left(z_{1}, z_{2}^{k}\right):=\left(\phi_{1}(z), \phi_{2}(z)\right)
$$

is a branched cover of order $k$, since

$$
\begin{aligned}
\left|\phi_{1}(z)\right|^{k}<\left|\phi_{2}(z)\right|<1 & \Longleftrightarrow\left|z_{1}\right|^{k}<\left|z_{2}^{k}\right|<1 \\
& \Longleftrightarrow\left|z_{1}\right|<\left|z_{2}\right|<1 .
\end{aligned}
$$

We note $u(z)=k z_{2}^{k-1}$, so $V$ is the set $\left\{z_{2}=0\right\}$, which is disjoint from $\mathbb{H}_{k}$. For each $j=1, \ldots, k$, the map $\Phi_{j}(z)=\left(z_{1}, \zeta^{j} z_{2}^{1 / k}\right)$ defines a local inverse of $\phi$, where $\zeta=e^{2 \pi i / k}$ and $z_{2}^{1 / k}$ is taken to mean the root with argument in the interval $[0,2 \pi / k)$. From this we see $U_{j}(z)=\left(\zeta^{j} z_{2}^{1 / k-1}\right) / k$. We now apply Bell's rule (3.2):

$$
\begin{aligned}
& \mathbb{B}_{k}\left(\left(z_{1}, z_{2}^{k}\right),\left(w_{1}, w_{2}\right)\right) \\
& =\frac{z_{2} \bar{w}_{2}^{1 / k}}{k^{2} z_{2}^{k} \bar{w}_{2}} \sum_{j=1}^{k} \mathbb{B}_{1}\left(\left(z_{1}, z_{2}\right),\left(w_{1}, \zeta^{j} w_{2}^{1 / k}\right)\right) \bar{\zeta}^{j} \\
& =\frac{z_{2}^{2} \bar{w}_{2}^{2 / k}}{\pi^{2} k^{2} z_{2}^{k} \bar{w}_{2}} \sum_{j=1}^{k} \frac{\bar{\zeta}^{2 j}}{\left(1-z_{2} \bar{w}_{2}^{1 / k} \bar{\zeta}^{j}\right)^{2}\left(z_{2} \bar{w}_{2}^{1 / k} \bar{\zeta}^{j}-z_{1} \bar{w}_{1}\right)^{2}} \\
& =\frac{a^{2-k}}{\pi^{2} k^{2}} \sum_{j=1}^{k} \frac{\bar{\zeta}^{2 j}}{\left(1-a \bar{\zeta}^{j}\right)^{2}\left(a \bar{\zeta}^{j}-s\right)^{2}},
\end{aligned}
$$

where $a=z_{2} \bar{w}_{2}^{1 / k}$ and $s=z_{1} \bar{w}_{1}$. Define $f_{j}(a, s):=\left(\zeta^{j}-a\right)^{2}\left(a-s \zeta^{j}\right)^{2}$ and notice that $\prod_{j=1}^{k} f_{j}(a, s)=\prod_{j=1}^{k}\left(\zeta^{j}-a\right)^{2} \cdot \prod_{j=1}^{k}\left(a-s \zeta^{j}\right)^{2}=\left(1-a^{k}\right)^{2}\left(a^{k}-s^{k}\right)^{2}$. 
Now, it follows that

$$
\text { (3.3) }=\frac{a^{2-k}}{\pi^{2} k^{2}} \sum_{j=1}^{k} \frac{\zeta^{2 j}}{f_{j}(a, s)}=\frac{a^{2-k} \sum_{j=1}^{k} F_{j}(a, s) \zeta^{2 j}}{\pi^{2} k^{2}\left(1-a^{k}\right)^{2}\left(a^{k}-s^{k}\right)^{2}},
$$

where $F_{j}(a, s):=\left(1-a^{k}\right)^{2}\left(a^{k}-s^{k}\right)^{2} / f_{j}(a, s)$. Notice each $F_{j}(a, s)$ can be written as a polynomial in $a$ of degree $4 k-4$, so the numerator of (3.4) takes the form

$$
a^{2-k} \sum_{j=1}^{k} F_{j}(a, s) \zeta^{2 j}=\sum_{j=2-k}^{3 k-2} g_{j}(s) a^{j}:=G(a, s) .
$$

We now wish to calculate the coefficient polynomials $g_{j}(s)$. Toward this goal, observe that $G\left(\zeta^{m} a, s\right)=G(a, s)$ for all $m \in \mathbb{Z}$. This follows because

$$
\begin{aligned}
G\left(\zeta^{m} a, s\right) & =\left(\zeta^{m} a\right)^{2-k} \sum_{j=1}^{k} F_{j}\left(\zeta^{m} a, s\right) \zeta^{2 j} \\
& =a^{2-k} \sum_{j=1}^{k} \frac{\left(1-a^{k}\right)^{2}\left(a^{k}-s^{k}\right)^{2}}{f_{j-m}(a, s)} \zeta^{2 j-2 m}=G(a, s) .
\end{aligned}
$$

Here, we've used the facts that

$$
f_{j}\left(\zeta^{m} a, s\right)=\zeta^{4 m} f_{j-m}(a, s) \quad \text { and } \quad f_{j}(a, s)=f_{j+m k}(a, s)
$$

for all $m \in \mathbb{Z}$. Because $G$ has this invariance, we conclude that

$$
G(a, s)=a^{2-k} \sum_{j=1}^{k} F_{j}(a, s) \zeta^{2 j}=g_{2 k}(s) a^{2 k}+g_{k}(s) a^{k}+g_{0}(s) .
$$

It remains to calculate $g_{2 k}(s), g_{k}(s)$ and $g_{0}(s)$, and these polynomials are obtained in the following lemma. But to avoid disrupting the flow of the paper with several pages of algebra, we postpone its proof until Section 5.

Lemma 3.7. The coefficient polynomials $g_{2 k}(s), g_{k}(s)$ and $g_{0}(s)$ are given by

$$
\begin{aligned}
g_{2 k}(s) & =k \sum_{l=1}^{k-1} l(k-l) s^{l-1}=k p_{k}(s), \\
g_{k}(s) & =k \sum_{l=1}^{k}\left(l^{2}+(k-l)^{2} s^{k}\right) s^{l-1}=k q_{k}(s), \\
g_{0}(s) & =k \sum_{l=1}^{k-1} l(k-l) s^{k+l-1}=k s^{k} p_{k}(s) .
\end{aligned}
$$

Using this lemma and letting $t:=a^{k}=z_{2}^{k} \bar{w}_{2}$, we see from (3.4) that 


$$
\mathbb{B}_{k}\left(\left(z_{1}, z_{2}^{k}\right),\left(w_{1}, w_{2}\right)\right)=\frac{p_{k}(s) t^{2}+q_{k}(s) t+s^{k} p_{k}(s)}{k \pi^{2}(1-t)^{2}\left(t-s^{k}\right)^{2}} .
$$

This is the desired formula for $\mathbb{B}_{k}$, except that both sides are a function of $z_{2}^{k}$. This is remedied by formally replacing the variable $z_{2}^{k}$ with $z_{2}$. This concludes the proof of Theorem 3.1

Remark 3.11. It's also true that the Bergman kernel of $\mathbb{U}_{m / n}$ is a rational function whenever $m, n \in \mathbb{Z}^{+}$. Indeed, the map $\left(z_{1}, z_{2}\right) \mapsto\left(z_{1} z_{2}^{n-1}, z_{2}^{m}\right)$ is a proper map from $\mathbb{H}_{1}$ onto $\mathbb{H}_{m / n}$, so Bell's formula gives $\mathbb{B}_{m / n}$ as a finite sum. Zapałowski [2016] characterizes the proper maps between fat Hartogs triangles. He shows there is a proper map $F: \mathbb{H}_{m / n} \rightarrow \mathbb{T}_{p / q}$ if and only if there are $a, b \in \mathbb{Z}^{+}$such that

$$
\frac{a q}{p}-\frac{b n}{m} \in \mathbb{Z} \text {. }
$$

Zapałowski's description of proper maps shows that the methods employed in this paper aren't able to say anything about fat Hartogs triangles $\mathbb{W}_{\gamma}$, for irrational $\gamma$.

Remark 3.12. Ramadanov's theorem says that if $\left\{\Omega_{k}\right\}$ is an increasing family of domains such that $\Omega_{k} \rightarrow \Omega \Subset \mathbb{C}^{n}$, then $\mathbb{B}_{\Omega_{k}}(z, w) \rightarrow \mathbb{B}_{\Omega}(z, w)$ absolutely and uniformly on compact subsets of $\Omega \times \Omega$. See [Ramadanov 1967] for the first appearance of this fact, and [Boas 1996] for a generalization in the smoothly bounded, pseudoconvex case. Notice that $\left\{\mathbb{W}_{k}\right\}$ is an increasing family and that $\mathbb{U}_{k} \rightarrow$ $D \times D^{*}$ as $k \rightarrow \infty$. Ramadanov's theorem shows that $\mathbb{B}_{k}(z, w) \rightarrow \mathbb{B}_{D \times D^{*}}(z, w)$, which is given in (2.5). This is difficult to see from direct computation.

Biholomorphism classes of domains. Let

$$
\psi(z)=\left(\psi_{1}(z), \psi_{2}(z)\right):=\left(z_{1} / z_{2}, z_{2}\right) .
$$

On page 330, we used the fact that $\psi: \mathbb{H}_{1} \rightarrow D \times D^{*}$ is a biholomorphism to compute the Bergman kernel of $\mathbb{H}_{1}$. We'll give a very similar argument to prove Theorem 1.4. Let $\Psi(z)=\left(z_{1} z_{2}, z_{2}\right)$, and see that $\Psi: D \times D^{*} \rightarrow \mathbb{H}_{1}$ is the inverse of $\psi$. Now, notice that $\psi: \mathbb{H}_{1 /(k+1)} \rightarrow \mathbb{M}_{1 / k}$ is also a biholomorphism for all $k \in \mathbb{Z}^{+}$, because

$$
\begin{aligned}
\left|\psi_{1}(z)\right|^{1 / k}<\left|\psi_{2}(z)\right|<1 & \Longleftrightarrow\left|\psi_{1}(z)\right|<\left|\psi_{2}(z)\right|^{k}<1 \\
& \Longleftrightarrow\left|z_{1} / z_{2}\right|<\left|z_{2}\right|^{k}<1 \\
& \Longleftrightarrow\left|z_{1}\right|<\left|z_{2}\right|^{k+1}<1 \\
& \Longleftrightarrow\left|z_{1}\right|^{1 /(k+1)}<\left|z_{2}\right|<1 .
\end{aligned}
$$

Let $\psi^{k}:=\psi \circ \cdots \circ \psi$ be $k$ copies of $\psi$ composed together, so $\psi^{k}(z):=\left(z_{1} z_{2}^{-k}, z_{2}\right)$. This gives a biholomorphism from $\mathbb{H}_{1 / k}$ to $D \times D^{*}$ with inverse $\Psi^{k}:=\left(z_{1} z_{2}^{k}, z_{2}\right)$. 
We illustrate this chain of biholomorphisms below:

$$
D \times D^{*} \underset{\psi}{\stackrel{\Psi}{\rightleftharpoons}} \mathbb{H}_{1} \underset{\psi}{\stackrel{\Psi}{\rightleftharpoons}} \mathbb{H}_{1 / 2} \underset{\psi}{\stackrel{\Psi}{\rightleftharpoons}} \cdots \underset{\psi}{\stackrel{\Psi}{\rightleftharpoons}} \mathbb{H}_{1 / k} \underset{\psi}{\stackrel{\Psi}{\rightleftharpoons}} \cdots .
$$

Proof of Theorem 1.4. Using the biholomorphism $\psi^{k}: \mathbb{H}_{1 / k} \rightarrow D \times D^{*}$ we easily obtain the desired formula. Indeed, since $\operatorname{det}\left[\left(\psi^{k}\right)^{\prime}\right](z)=z_{2}^{-k}$,

$$
\mathbb{B}_{1 / k}(z, w)=\frac{1}{z_{2}^{k} \bar{w}_{2}^{k}} \mathbb{B}_{1}\left(\psi_{k}(z), \psi_{k}(w)\right)=\frac{z_{2}^{k} \bar{w}_{2}^{k}}{\pi^{2}\left(1-z_{2} \bar{w}_{2}\right)^{2}\left(z_{2}^{k} \bar{w}_{2}^{k}-z_{1} \bar{w}_{1}\right)^{2}} .
$$

Remark 3.13. For $m, n \in \mathbb{Z}^{+}$, the map $\psi(z)=\left(z_{1} / z_{2}, z_{2}\right)$ also gives a biholomorphism from $\mathbb{U}_{m /(n+m)}$ onto $\mathbb{U}_{m / n}$. Applying this map recursively, we see that $\mathbb{U}_{m /(n+k m)}$ and $\mathbb{W}_{m / n}$ are biholomorphic for all $k \in \mathbb{Z}^{+}$.

\section{Consequences of the kernel formulas}

The Lu Qi-Keng problem. One of the long-standing open problems in Bergman theory is to classify the domains for which the Bergman kernel is nowhere vanishing. This question was first raised by Lu Qi-Keng [1966]. We say that a domain $\Omega \subset \mathbb{C}^{n}$ is Lu Qi-Keng when it has zero-free Bergman kernel, and the investigation of which domains have a zero-free Bergman kernel is known as the Lu Qi-Keng problem. See [Boas 2000] for a good historical survey, a few key points of which we now summarize.

The situation in the complex plane is relatively straightforward. When $\Omega \subset \mathbb{C}$ is simply connected, the Riemann mapping theorem together with (2.4) show that $\Omega$ is a Lu Qi-Keng domain, since the Bergman kernel of the unit disc is nonvanishing. But a finitely connected domain in $\mathbb{C}$ with at least two nonsingleton boundary components is not Lu Qi-Keng. See [Rosenthal 1969; Skwarczyński 1969] when $\Omega$ is an annulus, and [Bell 1992] for a more general class of domains.

There is no such simple characterization of the situation known in higher dimensions. In [Boas et al. 1999], it's shown there are smoothly bounded, strongly convex domains with real analytic boundary that are not Lu Qi-Keng in $\mathbb{C}^{n}$, when $n \geq 3$. Contrary to previous expectations, Boas [1996] shows that "most" pseudoconvex domains (with respect to a certain topology on the set of domains in $\mathbb{C}^{n}$ ) have vanishing Bergman kernel. Nevertheless, it is still desirable to understand why domains from certain classes have zero-free Bergman kernels, while domains from closely related classes may not. We now address this problem in the case of the domains $\mathbb{H}_{\gamma}$, where $\gamma \in \mathbb{Z}^{+}$and $\gamma^{-1} \in \mathbb{Z}^{+}$.

Using the explicit formulas for the Bergman kernels computed in the previous section, we can check whether or not these domains are Lu Qi-Keng. The following 
corollary is immediate from (1.5), whose numerator vanishes if and only if at least one of $z_{2}$ or $w_{2}$ equals zero.

Corollary 4.1. Let $k$ be a positive integer. The thin Hartogs triangle $\mathbb{H}_{1 / k}$ is a Lu Qi-Keng domain.

For fat Hartogs triangles with integer exponent $k \geq 2$, we deduce the following corollary from (1.3).

Corollary 4.2. Let $k \geq 2$ be an integer. The fat Hartogs triangle $\mathbb{H}_{k}$ is not a Lu Qi-Keng domain.

Proof. First consider the case $k \geq 3$. Let $z=(0, i / \sqrt{k-1})$ and $w=(0,-i / \sqrt{k-1})$. Then $z, w \in \mathbb{H}_{k}$. Since $p_{k}(0)=k-1$ and $q_{k}(0)=1$, we see that $\mathbb{B}_{k}(z, w)=0$. When $k=2$, let $z=(i / \sqrt{2},(\sqrt{7}+i) / 4)$ and $w=(-i / \sqrt{2},(\sqrt{7}-i) / 4)$. It is easily checked that $z, w \in \mathbb{H}_{2}$ and that $\mathbb{B}_{2}(z, w)=0$.

It's immediate from (2.4) that a nonvanishing Bergman kernel is a biholomorphic invariant. Corollary 4.2 lets us deduce the following:

Corollary 4.3. Let $k \geq 2$ be an integer. $\mathbb{H}_{k}$ is not biholomorphic to $D \times D^{*}$.

Remark 4.4. Using Ramadanov's theorem in conjunction with Hurwitz's theorem on zeroes of holomorphic functions, we see that for each integer $k \geq 2$, there is an $s_{k} \in[k-1, k)$ such that for all $\gamma \in\left(s_{k}, k\right]$, the Bergman kernel $\mathbb{B}_{\gamma}$ of $\mathbb{U}_{\gamma}$ has zeroes. It seems plausible to conjecture that $s_{k}=k-1$, i.e., that no fat Hartogs triangle of exponent $\gamma>1$ is Lu Qi-Keng.

Remark 4.5. As was mentioned in Remark 3.12, $\mathbb{H}_{k} \rightarrow D \times D^{*}$ as $k \rightarrow \infty$. The Bergman kernel $\mathbb{B}_{D \times D^{*}}$ is zero free, so for any fixed compact subset $K \subset D \times D^{*}$, Ramadanov's theorem tells us that the Bergman kernel $\mathbb{B}_{k}$ restricted to $K$ is zero free for all $k$ sufficiently large. We see this happen as the zero of $\mathbb{B}_{k}$ provided in the proof of Corollary 4.2 is pushed to the origin. It would be interesting to do further analysis of the zero set of the polynomial in the numerator of $\mathbb{B}_{k}$.

Diagonal boundary behavior. The asymptotic behavior of $\mathbb{B}_{\Omega}(z, z)$ as $z$ tends to the boundary has been studied for many classes of smoothly bounded, pseudoconvex domains. [Hörmander 1965; Fefferman 1974] are two seminal papers dealing with the strongly pseudoconvex case. Results also exist for many classes of smoothly bounded, weakly pseudoconvex domains. See [McNeal 1989; Catlin 1989; Nagel et al. 1989] for finite-type domains in $\mathbb{C}^{2}$, and [McNeal 1994] for finite-type, convex domains in $\mathbb{C}^{n}$. Refer to [Fu 2014] for analogous results on smoothly bounded domains with constant Levi rank. But all these estimates are for classes of domains with boundary smoothness, and there are presently no general theorems about the behavior of $\mathbb{B}_{\Omega}(z, z)$ for pseudoconvex domains near singular boundary points.

Using the explicit formulas for the Bergman kernel, we establish the following: 
Lemma 4.6. Let $k \in \mathbb{Z}^{+}$. We have the following behavior of the Bergman kernel restricted to the diagonal:

$$
\mathbb{B}_{k}(z, z) \approx \frac{1}{\left(1-\left|z_{2}\right|\right)^{2}\left(\left|z_{2}\right|-\left|z_{1}\right|^{k}\right)^{2}}, \quad z \in \mathbb{H}_{k} .
$$

Proof. In this proof we are concerned with $\mathbb{B}_{k}(z, z)$, so write $s:=\left|z_{1}\right|^{2}$ and $t:=\left|z_{2}\right|^{2}$. From Theorem 1.2 we see that

$$
\mathbb{B}_{k}(z, z)=\frac{p_{k}(s) t^{2}+q_{k}(s) t+s^{k} p_{k}(s)}{k \pi^{2}(1-t)^{2}\left(t-s^{k}\right)^{2}},
$$

where $p_{k}(s)$ and $q_{k}(s)$ are given in the statement of Theorem 1.2. We now estimate the numerator of (4.8). Notice that $q_{k}(s) \geq 1$ for all $s \in[0,1)$, and so

$$
t \leq p_{k}(s) t^{2}+q_{k}(s) t+s^{k} p_{k}(s)<t\left[2 p_{k}(1)+q_{k}(1)\right] \lesssim t,
$$

since $s^{k}<t$. Now estimate the terms in the denominator. It's easy to see that both

$$
\begin{aligned}
(1-t)^{2} & \approx\left(1-\left|z_{2}\right|\right)^{2}, \\
\left(t-s^{k}\right)^{2} & \approx\left|z_{2}\right|^{2}\left(\left|z_{2}\right|-\left|z_{1}\right|^{k}\right)^{2} .
\end{aligned}
$$

Here, we've used the fact that $\left|z_{2}\right|^{2} \leq\left(\left|z_{2}\right|+\left|z_{1}\right|^{k}\right)^{2}<4\left|z_{2}\right|^{2}$. Putting these estimates together, we obtain (4.7).

Let $\Omega \subset \mathbb{C}^{2}$ be a bounded domain and $\zeta \in b \Omega$ a smooth, Levi-flat boundary point. It can be shown that $\mathbb{B}_{\Omega}(z, z) \approx \delta_{\Omega}(z)^{-2}$ as $z \rightarrow \zeta$. See [Fu 2014] for more information. The domains $\mathbb{H}_{k}$ are Levi-flat at all smooth boundary points, because the smooth parts of the boundary can be locally foliated by analytic discs. We explicitly see this asymptotic behavior from estimate (4.7). In fact, this estimate also lets us determine the asymptotic growth rate of $\mathbb{B}_{k}(z, z)$ as $z$ tends to the boundary singularity at the origin. When $z$ is sufficiently close to 0 , it's straightforward to see $\left|z_{2}\right|-\left|z_{1}\right|^{k} \approx \delta_{k}(z)$, the distance of $z$ to the boundary of $\mathbb{H}_{k}$. From this, we deduce:

Theorem 4.9. Let $k \in \mathbb{Z}^{+}$and $\delta_{k}(z)$ be the distance of $z$ to $b \boxplus_{k}$. Then

$$
\mathbb{B}_{k}(z, z) \approx \delta_{k}(z)^{-2} \text { as } z \rightarrow 0 .
$$

Remark 4.10. Following steps analogous to those in Lemma 4.6, we can show

$$
\mathbb{B}_{1 / k}(z, z) \approx \frac{1}{\left(1-\left|z_{2}\right|\right)^{2}\left(\left|z_{2}\right|^{k}-\left|z_{1}\right|\right)^{2}}, \quad z \in \mathbb{M}_{1 / k} .
$$

This estimate can be used to determine the asymptotic growth rate of $\mathbb{B}_{1 / k}(z, z)$ as $z$ tends to the boundary singularity at the origin. When $z$ is sufficiently close to 0 , it's straightforward to check that $\left|z_{2}\right|^{k}-\left|z_{1}\right| \approx \delta_{1 / k}(z)$, the distance of $z$ to $b \mathbb{1}_{1 / k}$. From this we conclude that $\mathbb{B}_{1 / k}(z, z) \approx \delta_{1 / k}(z)^{-2}$ as $z \rightarrow 0$. 


\section{Proof of Lemma 3.7}

Equation (3.6) tells us that

$$
a^{2-k} \sum_{j=1}^{k} F_{j}(a, s) \zeta^{2 j}=g_{2 k}(s) a^{2 k}+g_{k}(s) a^{k}+g_{0}(s) .
$$

We prove Lemma 3.7 by splitting the calculation of $g_{2 k}(s), g_{k}(s)$ and $g_{0}(s)$ into two separate lemmas.

Lemma 5.2. Let

$$
h_{l}(s):=\sum_{r=0}^{l} s^{r} .
$$

For each $j=1, \ldots, k$, the respective coefficient functions of the $a^{3 k-2}, a^{2 k-2}$ and $a^{k-2}$ terms of $F_{j}(a, s) \zeta^{2 j}$ are equal to the following:

$$
\begin{aligned}
a^{3 k-2}: & \sum_{l=0}^{k-2} h_{l}(s) h_{k-2-l}(s), \\
a^{2 k-2}: & 2 \sum_{l=0}^{k-2} s^{k-1-l} h_{l}(s)^{2}+h_{k-1}(s)^{2}, \\
a^{k-2}: & s^{k} \sum_{l=0}^{k-2} h_{l}(s) h_{k-2-l}(s) .
\end{aligned}
$$

In particular, note that these expressions have no $j$ dependence.

Proof. In this calculation of the coefficient functions of the $a^{3 k-2}, a^{2 k-2}$ and $a^{k-2}$ terms appearing in $F_{j}(a, s) \zeta^{2 j}$, we'll often write $\theta:=\zeta^{j}$ to cut down on superscripts.

$$
\begin{aligned}
F_{j}(a, s) & =\frac{\left(1-a^{k}\right)^{2}\left(a^{k}-s^{k}\right)^{2}}{f_{j}(a, s)}=\left(\frac{a^{k}-1}{a-\theta}\right)^{2}\left(\frac{a^{k}-s^{k}}{a-s \theta}\right)^{2} \\
& =\left(\sum_{m=1}^{k} a^{k-m} \theta^{m-1}\right)^{2}\left(\sum_{n=1}^{k} a^{k-n}(s \theta)^{n-1}\right)^{2} \\
& =\left(\sum_{m=1}^{k} \sum_{n=1}^{k} a^{2 k-m-n} \theta^{m+n-2} s^{n-1}\right)^{2} .
\end{aligned}
$$

To better understand the double sum inside the parentheses of (5.3) above, we split this sum into three pieces, $A, B$ and $C$, depending on the value of $m+n$. Let $A$ be the sum of the terms with $2 \leq m+n \leq k, B$ be the sum of the terms with $m+n=k+1$, and $C$ be the sum of the terms with $k+2 \leq m+n \leq 2 k$. 
We rewrite $A$ by letting $l=m+n-2$ be the index of summation. Then

$$
A=\sum_{l=0}^{k-2} a^{2 k-l-2} \theta^{l} h_{l}(s) .
$$

For $B$, only include those terms with $m+n=k+1$, so we don't have an outside sum. Therefore,

$$
B=a^{k-1} \theta^{k-1} h_{k-1}(s) .
$$

For $C$, let $l=m+n-k-2$ be the index of summation. Then

$$
C=\sum_{l=0}^{k-2} a^{k-2-l} \theta^{k+l} s^{l+1} h_{k-2-l}(s) .
$$

So we have

$$
\begin{aligned}
(5.3) & =\left(\sum_{l=0}^{k-2} a^{2 k-2-l} \theta^{l} h_{l}(s)+a^{k-1} \theta^{k-1} h_{k-1}(s)+\sum_{l=0}^{k-2} a^{k-2-l} \theta^{k+l} s^{l+1} h_{k-2-l}(s)\right)^{2} \\
& =(A+B+C)^{2} \\
& =A^{2}+B^{2}+C^{2}+2 A B+2 B C+2 A C .
\end{aligned}
$$

I emphasize that as a polynomial in $a, A$ has powers of $a$ ranging from $a^{2 k-2}$ to $a^{k}, B$ only has an $a^{k-1}$ term, and $C$ has terms ranging from $a^{k-2}$ to $a^{0}$. This observation greatly simplifies the computations below.

Computation of the $\boldsymbol{a}^{3 k-2}$ coefficient. For the coefficient of the $a^{3 k-2}$ term in $F_{j}(a, s) \theta^{2}$, it is sufficient to consider the coefficient function of $a^{3 k-2}$ in $A^{2} \theta^{2}$ :

$$
\begin{aligned}
A^{2} \theta^{2} & =\left(\sum_{m=0}^{k-2} a^{2 k-2-m} \theta^{m} h_{m}(s)\right)\left(\sum_{n=0}^{k-2} a^{2 k-2-n} \theta^{n} h_{n}(s)\right) \theta^{2} \\
& =\theta^{2} \sum_{m=0}^{k-2} \sum_{n=0}^{k-2} a^{4 k-4-m-n} \theta^{m+n} h_{m}(s) h_{n}(s) .
\end{aligned}
$$

Letting $m+n=k-2$, we find that the coefficient function of $a^{3 k-2}$ is independent of $\theta$ (since $\theta^{k}=1$ ), and therefore independent of $j$. This function is given by

$$
\sum_{l=0}^{k-2} h_{l}(s) h_{k-2-l}(s) \text {. }
$$

Computation of the $\boldsymbol{a}^{\mathbf{2 k - 2}}$ coefficient. For the coefficient of the $a^{2 k-2}$ term in $F_{j}(a, s) \theta^{2}$, it is sufficient to consider the coefficient of $a^{2 k-2}$ in $\left(2 A C+B^{2}\right) \theta^{2}$ : 


$$
\begin{aligned}
& \left(2 A C+B^{2}\right) \theta^{2} \\
& =\left[2\left(\sum_{m=0}^{k-2} a^{2 k-2-m} \theta^{m} h_{m}(s)\right)\left(\sum_{n=0}^{k-2} a^{k-2-n} \theta^{k+n} s^{n+1} h_{k-2-n}(s)\right)\right. \\
& =\left[2 \sum_{m=0}^{k-2} \sum_{n=0}^{k-2} a^{3 k-4-m-n} \theta^{k+m+n} s^{n+1} h_{m}(s) h_{k-2-n}(s)+a^{2 k-2} \theta^{2 k-2} h_{k-1}(s)^{2}\right] \theta^{2} .
\end{aligned}
$$

Letting $m+n=k-2$, we find that the coefficient function of $a^{2 k-2}$ is independent of $\theta$ (since $\theta^{2 k}=1$ ), and therefore independent of $j$. This function is given by

$$
2 \sum_{l=0}^{k-2} s^{k-1-l} h_{l}(s)^{2}+h_{k-1}(s)^{2} .
$$

Computation of the $a^{k-2}$ coefficient. For the coefficient of the $a^{k-2}$ term, it is sufficient to determine the coefficient of $a^{k-2}$ in $C^{2} \theta^{2}$ :

$$
\begin{aligned}
C^{2} \theta^{2} & =\left(\sum_{m=0}^{k-2} a^{k-2-m} \theta^{k+m} s^{m+1} h_{k-2-m}(s)\right)\left(\sum_{n=0}^{k-2} a^{k-2-n} \theta^{k+n} s^{n+1} h_{k-2-n}(s)\right) \theta^{2} \\
& =\theta^{2} \sum_{m=0}^{k-2} \sum_{n=0}^{k-2} a^{2 k-4-m-n} \theta^{2 k+m+n} s^{m+n+2} h_{k-2-m}(s) h_{k-2-n}(s) .
\end{aligned}
$$

Letting $m+n=k-2$, we find that the coefficient function of $a^{k-2}$ is independent of $\theta$ (since $\theta^{3 k}=1$ ), and therefore independent of $j$. This function is given by

$$
s^{k} \sum_{l=0}^{k-2} h_{l}(s) h_{k-2-l}(s) .
$$

Now we rewrite (5.4), (5.5) and (5.6) as simpler polynomials:

Lemma 5.7. Again, let $h_{l}(s)=\sum_{r=0}^{l} s^{r}$. Then we have the following equalities:

$$
\begin{gathered}
\sum_{l=0}^{k-2} h_{l}(s) h_{k-2-l}(s)=\sum_{l=1}^{k-1} l(k-l) s^{l-1}, \\
2 \sum_{l=0}^{k-2} s^{k-1-l} h_{l}(s)^{2}+h_{k-1}(s)^{2}=\sum_{l=1}^{k}\left(l^{2}+(k-l)^{2} s^{k}\right) s^{l-1} .
\end{gathered}
$$

Proof. Focus on (5.8) first. Notice that

$$
h_{l}(s) h_{k-2-l}(s)=\left(\sum_{m=0}^{l} s^{m}\right)\left(\sum_{n=0}^{k-2-l} s^{n}\right)=\sum_{r=0}^{k-2} s^{r}+\sum_{r=1}^{k-3} s^{r}+\cdots+\sum_{r=L}^{k-2-L} s^{r},
$$


where $L=\min \{l, k-2-l\}$. Using this, we see

$$
\begin{aligned}
(5.4) & =\sum_{l=0}^{k-2} h_{l}(s) h_{k-2-l}(s) \\
& =\sum_{l=0}^{k-2}\left(\sum_{r=0}^{k-2} s^{r}+\sum_{r=1}^{k-3} s^{r}+\cdots+\sum_{r=L}^{k-2-L} s^{r}\right) \\
& =(k-1) \sum_{r=0}^{k-2} s^{r}+(k-3) \sum_{r=1}^{k-3} s^{r}+\cdots+(k-2 K-1) \sum_{r=K}^{k-2-K} s^{r},
\end{aligned}
$$

where $K=\left\lfloor\frac{1}{2}(k-2)\right\rfloor$. From here, we compute that the coefficient of $s^{l}$ in (5.4) is given by

$$
\begin{aligned}
\sum_{m=0}^{L}(k-2 m-1) & =(L+1)(k-1)-2 \sum_{m=0}^{L} m \\
& =(L+1)(k-L-1)=(l+1)(k-l-1) .
\end{aligned}
$$

Therefore,

$$
(5.4)=\sum_{l=0}^{k-2} h_{l}(s) h_{k-2-l}(s)=\sum_{l=0}^{k-2}(l+1)(k-l-1) s^{l}=\sum_{l=1}^{k-1} l(k-l) s^{l-1},
$$

where we've re-indexed the sum in the last equality, obtaining the form of (5.8).

Now we'll establish (5.9). Note that

$$
h_{r}(s)^{2}=1+2 s+\cdots+r s^{r-1}+(r+1) s^{r}+r s^{r+1}+\cdots+2 s^{2 r-1}+s^{2 r} .
$$

Using this, write the pieces of $(5.5)=2 \sum_{l=0}^{k-2} s^{k-1-l} h_{l}(s)^{2}+h_{k-1}(s)^{2}$ in the following way:

$$
\begin{array}{cc}
s^{k-1} h_{0}(s)^{2}= & s^{k-1} \\
s^{k-2} h_{1}(s)^{2}= & s^{k-2}+2 s^{k-1}+s^{k} \\
s^{k-3} h_{2}(s)^{2}= & s^{k-3}+2 s^{k-2}+3 s^{k-1}+2 s^{k}+s^{k+1} \\
\vdots & \vdots \\
h_{k-1}(s)^{2}=1+\cdots+(k-1) s^{k-2}+k s^{k-1}+(k-1) s^{k}+\cdots+s^{2 k-2} .
\end{array}
$$

The coefficient of $s^{l}$ in (5.5) can be obtained by considering the vertical columns above. Notice the coefficients of $s^{l}$ and $s^{2 k-2-l}$ are always the same. When $0 \leq l \leq k-1$, we have that the coefficient of $s^{l}$ is given by

$$
2 \sum_{r=1}^{l} r+(l+1)=(l+1)^{2}
$$


Therefore,

$$
\begin{aligned}
(5.5) & =\sum_{l=0}^{k-1}(l+1)^{2} s^{l}+\sum_{l=0}^{k-2}(k-(l+1))^{2} s^{k+l} \\
& =\sum_{l=0}^{k-1}\left((l+1)^{2}+(k-l-1)^{2} s^{k}\right) s^{l} \\
& =\sum_{l=1}^{k}\left(l^{2}+(k-l)^{2} s^{k}\right) s^{l-1},
\end{aligned}
$$

where we have re-indexed the sum in the last equality to obtain the form of (5.9).

Proof of Lemma 3.7. Lemmas 5.2 and 5.7 together give us (3.8), (3.9) and (3.10).

\section{Acknowledgements}

I would like to thank my advisor, Jeff McNeal, for introducing me to Bergman theory, and for his ongoing support and encouragement. The many interesting discussions in his office have contributed to my growth, both as a mathematician and a human being. I would also like to thank the anonymous referee who offered many useful suggestions to improve the original draft of this paper.

\section{References}

[Bell 1982] S. R. Bell, "The Bergman kernel function and proper holomorphic mappings", Trans. Amer. Math. Soc. 270:2 (1982), 685-691. MR Zbl

[Bell 1992] S. R. Bell, The Cauchy transform, potential theory, and conformal mapping, CRC Press, Boca Raton, FL, 1992. MR

[Boas 1996] H. P. Boas, "The Lu Qi-Keng conjecture fails generically", Proc. Amer. Math. Soc. 124:7 (1996), 2021-2027. MR Zbl

[Boas 2000] H. P. Boas, "Lu Qi-Keng's problem”, J. Korean Math. Soc. 37:2 (2000), 253-267. MR $\mathrm{Zbl}$

[Boas et al. 1999] H. P. Boas, S. Fu, and E. J. Straube, "The Bergman kernel function: Explicit formulas and zeroes", Proc. Amer. Math. Soc. 127:3 (1999), 805-811. MR Zbl

[Bremermann 1955] H. J. Bremermann, "Holomorphic continuation of the kernel function and the Bergman metric in several complex variables", pp. 349-383 in Lectures on functions of a complex variable, edited by W. Kaplan, The University of Michigan Press, Ann Arbor, 1955. MR Zbl

[Catlin 1989] D. W. Catlin, "Estimates of invariant metrics on pseudoconvex domains of dimension two", Math. Z. 200:3 (1989), 429-466. MR Zbl

[Chakrabarti and Shaw 2013] D. Chakrabarti and M.-C. Shaw, "Sobolev regularity of the $\bar{\partial}$-equation on the Hartogs triangle", Math. Ann. 356:1 (2013), 241-258. MR Zbl

[Chakrabarti and Zeytuncu 2016] D. Chakrabarti and Y. E. Zeytuncu, " $L$ p mapping properties of the Bergman projection on the Hartogs triangle”, Proc. Amer. Math. Soc. 144:4 (2016), 1643-1653. MR Zbl 
[Chen 2013] L. Chen, "The $L^{p}$ boundedness of the Bergman projection for a class of bounded Hartogs domains", preprint, 2013. arXiv 1304.7898

[D'Angelo 1978] J. P. D'Angelo, “A note on the Bergman kernel”, Duke Math. J. 45:2 (1978), 259-265. MR Zbl

[D’Angelo 1994] J. P. D’Angelo, "An explicit computation of the Bergman kernel function", J. Geom. Anal. 4:1 (1994), 23-34. MR Zbl

[Edholm and McNeal 2016] L. D. Edholm and J. D. McNeal, "The Bergman projection on fat Hartogs triangles: $L^{p}$ boundedness", Proc. Amer. Math. Soc. 144:5 (2016), 2185-2196. MR Zbl

[Fefferman 1974] C. Fefferman, "The Bergman kernel and biholomorphic mappings of pseudoconvex domains”, Invent. Math. 26 (1974), 1-65. MR Zbl

[Fu 2014] S. Fu, "Estimates of invariant metrics on pseudoconvex domains near boundaries with constant Levi ranks", J. Geom. Anal. 24:1 (2014), 32-46. MR Zbl

[Hörmander 1965] L. Hörmander, " $L^{2}$ estimates and existence theorems for the $\bar{\partial}$ operator", Acta Math. 113 (1965), 89-152. MR Zbl

[Krantz 1992] S. G. Krantz, Function theory of several complex variables, 2nd ed., Wadsworth \& Brooks/Cole, Pacific Grove, CA, 1992. MR Zbl

[Lu 1966] Q.-K. Lu, “On Kaehler manifolds with constant curvature”, Chinese Math.-Acta 8 (1966), 283-298. MR Zbl

[McNeal 1989] J. D. McNeal, "Boundary behavior of the Bergman kernel function in $\mathbf{C}^{2}$ ", Duke Math. J. 58:2 (1989), 499-512. MR Zbl

[McNeal 1994] J. D. McNeal, "Estimates on the Bergman kernels of convex domains", Adv. Math. 109:1 (1994), 108-139. MR Zbl

[Nagel et al. 1989] A. Nagel, J.-P. Rosay, E. M. Stein, and S. Wainger, "Estimates for the Bergman and Szegő kernels in $\mathbf{C}^{2}$ ", Ann. of Math. (2) 129:1 (1989), 113-149. MR Zbl

[Park 2008] J.-D. Park, "New formulas of the Bergman kernels for complex ellipsoids in $\mathbb{C}^{2}$, Proc. Amer. Math. Soc. 136:12 (2008), 4211-4221. MR Zbl

[Ramadanov 1967] I. Ramadanov, "Sur une propriété de la fonction de Bergman", C. R. Acad. Bulgare Sci. 20 (1967), 759-762. MR Zbl

[Rosenthal 1969] P. Rosenthal, "On the zeros of the Bergman function in doubly-connected domains", Proc. Amer. Math. Soc. 21 (1969), 33-35. MR Zbl

[Skwarczyński 1969] M. Skwarczyński, "The invariant distance in the theory of pseudoconformal transformations and the Lu Qi-Keng conjecture", Proc. Amer. Math. Soc. 22 (1969), 305-310. MR $\mathrm{Zbl}$

[Zapałowski 2016] P. Zapałowski, "Proper holomorphic mappings between generalized Hartogs triangles”, preprint, 2016. arXiv 1601.01806

Received June 27, 2015. Revised February 26, 2016.

LUKE D. EDHOLM

DEPARTMENT OF MATHEMATICS

The OHIo State University

COLumbus, OH 43201

UNITED STATES

edholm.1@osu.edu 


\title{
PACIFIC JOURNAL OF MATHEMATICS
}

Founded in 1951 by E. F. Beckenbach (1906-1982) and F. Wolf (1904-1989)

$$
\text { msp.org/pjm }
$$

\section{EDITORS}

\author{
Don Blasius (Managing Editor) \\ Department of Mathematics \\ University of California \\ Los Angeles, CA 90095-1555 \\ blasius@math.ucla.edu
}

\author{
Paul Balmer \\ Department of Mathematics \\ University of California \\ Los Angeles, CA 90095-1555 \\ balmer@math.ucla.edu \\ Robert Finn \\ Department of Mathematics \\ Stanford University \\ Stanford, CA 94305-2125 \\ finn@math.stanford.edu \\ Sorin Popa \\ Department of Mathematics \\ University of California \\ Los Angeles, CA 90095-1555 \\ popa@math.ucla.edu
}

\author{
Vyjayanthi Chari \\ Department of Mathematics \\ University of California \\ Riverside, CA 92521-0135 \\ chari@math.ucr.edu \\ Kefeng Liu \\ Department of Mathematics \\ University of California \\ Los Angeles, CA 90095-1555 \\ liu@math.ucla.edu \\ Igor Pak \\ Department of Mathematics \\ University of California \\ Los Angeles, CA 90095-1555 \\ pak.pjm@gmail.com \\ Paul Yang \\ Department of Mathematics \\ Princeton University \\ Princeton NJ 08544-1000 \\ yang@math.princeton.edu
}

\section{PRODUCTION}

Silvio Levy, Scientific Editor, production@msp.org

\section{SUPPORTING INSTITUTIONS}

ACADEMIA SINICA, TAIPEI

CALIFORNIA INST. OF TECHNOLOGY

STANFORD UNIVERSITY

UNIV. OF BRITISH COLUMBIA

UNIV. OF CALIFORNIA, BERKELEY

UNIV. OF CALIFORNIA, DAVIS

UNIV. OF CALIFORNIA, LOS ANGELES

UNIV. OF CALIFORNIA, RIVERSIDE

UNIV. OF CALIFORNIA, SAN DIEGO

UNIV. OF CALIF., SANTA BARBARA
KEIO UNIVERSITY

MATH. SCIENCES RESEARCH INSTITUTE

NEW MEXICO STATE UNIV.

OREGON STATE UNIV.
Daryl Cooper

Department of Mathematics

University of California

Santa Barbara, CA 93106-3080 cooper@math.ucsb.edu

Jiang-Hua Lu

Department of Mathematics

The University of Hong Kong

Pokfulam Rd., Hong Kong

jhlu@maths.hku.hk

$$
\text { Jie Qing }
$$

Department of Mathematics

University of California

Santa Cruz, CA 95064

qing@ cats.ucsc.edu

\author{
UNIV. OF CALIF., SANTA CRUZ \\ UNIV. OF MONTANA \\ UNIV. OF OREGON \\ UNIV. OF SOUTHERN CALIFORNIA \\ UNIV. OF UTAH \\ UNIV. OF WASHINGTON \\ WASHINGTON STATE UNIVERSITY
}

These supporting institutions contribute to the cost of publication of this Journal, but they are not owners or publishers and have no responsibility for its contents or policies.

See inside back cover or msp.org/pjm for submission instructions.

The subscription price for 2016 is US $\$ 440 /$ year for the electronic version, and \$600/year for print and electronic.

Subscriptions, requests for back issues and changes of subscriber address should be sent to Pacific Journal of Mathematics, P.O. Box 4163, Berkeley, CA 94704-0163, U.S.A. The Pacific Journal of Mathematics is indexed by Mathematical Reviews, Zentralblatt MATH, PASCAL CNRS Index, Referativnyi Zhurnal, Current Mathematical Publications and Web of Knowledge (Science Citation Index).

The Pacific Journal of Mathematics (ISSN 0030-8730) at the University of California, c/o Department of Mathematics, 798 Evans Hall \#3840, Berkeley, CA 94720-3840, is published twelve times a year. Periodical rate postage paid at Berkeley, CA 94704, and additional mailing offices. POSTMASTER: send address changes to Pacific Journal of Mathematics, P.O. Box 4163, Berkeley, CA 94704-0163.

PJM peer review and production are managed by EditFLOW ${ }^{\circledR}$ from Mathematical Sciences Publishers.

PUBLISHED BY

\section{I. mathematical sciences publishers}

nonprofit scientific publishing

http://msp.org/

(C) 2016 Mathematical Sciences Publishers 


\section{PACIFIC JOURNAL OF MATHEMATICS}

Volume $284 \quad$ No. $2 \quad$ October 2016

Spherical CR Dehn surgeries

Miguel ACOsta

Degenerate flag varieties and Schubert varieties: a characteristic free approach

283

Giovanni Cerulli Irelli, Martina LANini and Peter

LITTELMANN

Solitons for the inverse mean curvature flow

Gregory Drugan, HoJoo LEE and GLEN WHEELER

Bergman theory of certain generalized Hartogs triangles

\section{LUKE D. EDHOLM}

Transference of certain maximal Hilbert transforms on the torus

DASHAN FAN, HUOXIONG WU and FAYOU ZHAO

The Turaev and Thurston norms

STEFAN FriedL, DANIEL S. Silver and Susan G. WiLLiams

A note on nonunital absorbing extensions

JAMES GABE

On nonradial singular solutions of supercritical biharmonic equations

Zongming GuO, JUNCHENG WeI and Wen YANG

Natural commuting of vanishing cycles and the Verdier dual

\section{DAVID B. MASSEY}

The nef cones of and minimal-degree curves in the Hilbert schemes of points on certain surfaces

ZHENBo QIN and YUPING TU

Smooth approximation of conic Kähler metric with lower Ricci curvature bound

\section{LIANGMING SHEN}

Maps from the enveloping algebra of the positive Witt algebra to regular algebras

Susan J. SierRa and Chelsea Walton 